JOURNAL OF

APPLIED

CRYSTALLOGRAPHY

ISSN 1600-5767

Received 24 January 2016

Accepted 3 February 2016

Keywords: diffuse scattering; quantitative analysis; supercomputers.

\section{Analyzing diffuse scattering with supercomputers. Corrigendum}

\author{
T. M. Michels-Clark, ${ }^{a, b} *$ V. E. Lynch, ${ }^{b}$ C. M. Hoffmann, ${ }^{b}$ J. Hauser, ${ }^{c}$ T. Weber, \\ R. Harrison ${ }^{e}$ and H. B. Bürgi ${ }^{c, f}$
}

aLawrence Berkeley National Laboratory, Berkeley, CA 94720, USA, 'bak Ridge National Laboratory, PO Box 2008, Oak Ridge, TN 37831, USA, ' Department of Chemistry and Biochemistry, University of Bern, Freiestrasse 3, CH-3012, Bern, Switzerland, $\mathbf{d}_{\mathrm{ETH}}$ Zürich, Laboratorium für Kristallographie, Wolfgang-Pauli-Strasse 10, 8093 Zürich, Switzerland, ${ }^{\mathbf{e}}$ Stony Brook University, Department of Applied Mathematics and Statistics, Stony Brook, NY 11794-3600, USA, and fDepartment of Chemistry, University of Zürich, Winterthurerstrasse 190, CH-8057 Zürich, Switzerland. *Correspondence e-mail: tmichels-clark@ion.chem.utk.edu

Errors in the paper by Michels-Clark, Lynch, Hoffmann, Hauser, Weber, Harrison \& Bürgi [J. Appl. Cryst. (2013), 46, 1616-1625] are corrected.

The paper by Michels-Clark et al. (2013) contains misleading errors which are corrected here. The numerical results reported in that paper and the conclusions given there are not
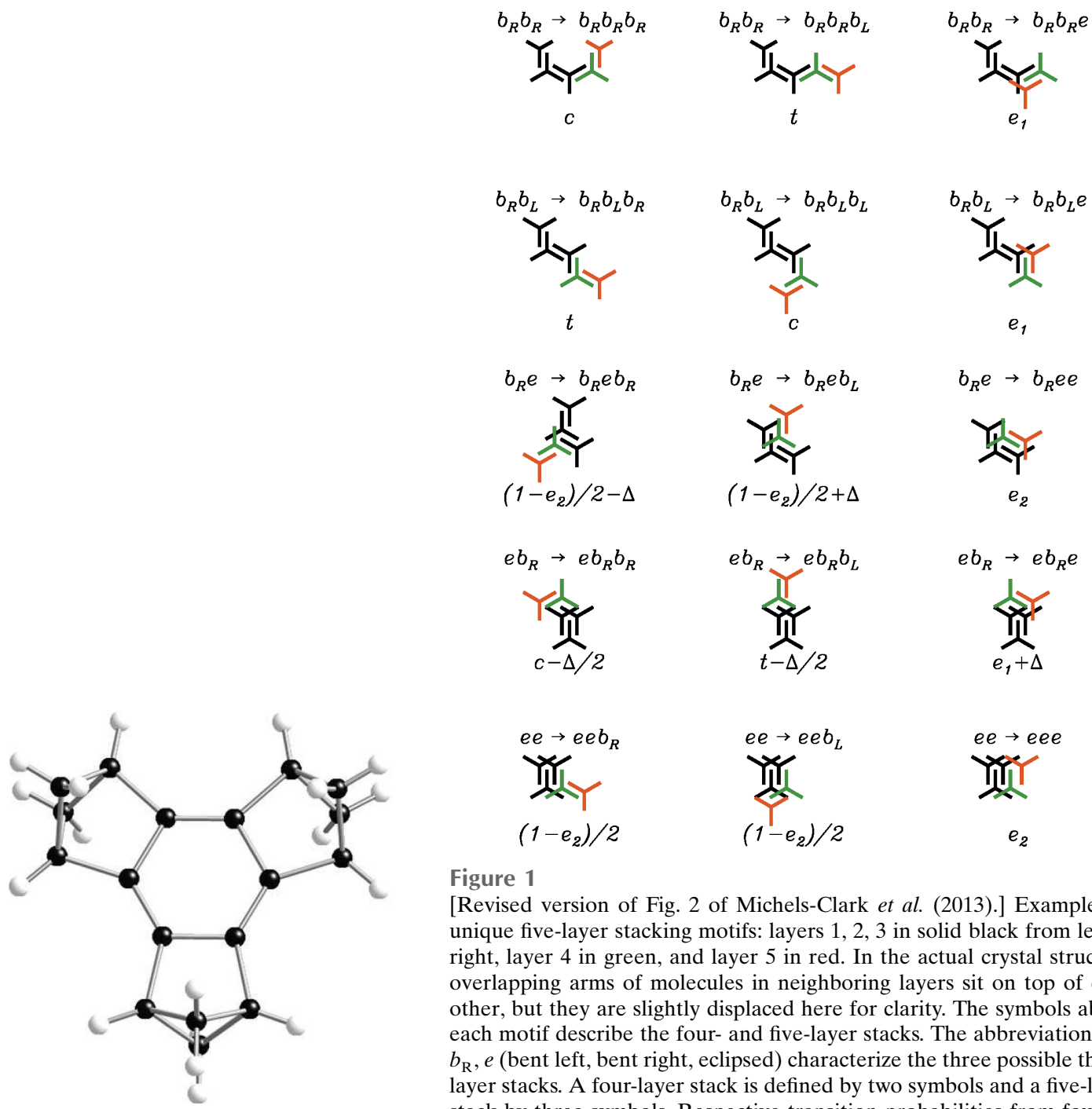

Figure 1

[Revised version of Fig. 2 of Michels-Clark et al. (2013).] Examples of unique five-layer stacking motifs: layers 1,2,3 in solid black from left to right, layer 4 in green, and layer 5 in red. In the actual crystal structure overlapping arms of molecules in neighboring layers sit on top of each other, but they are slightly displaced here for clarity. The symbols above each motif describe the four- and five-layer stacks. The abbreviations $b_{\mathrm{L}}$, $b_{\mathrm{R}}, e$ (bent left, bent right, eclipsed) characterize the three possible threelayer stacks. A four-layer stack is defined by two symbols and a five-layer stack by three symbols. Respective transition probabilities from four- to five-layer stackings are given at the bottom of each motif. 
Table 1

(Revised) The transition matrix $\mathbf{T}$ of probabilities for extending the left-hand column of four-layer motifs into the top row of new four-layer motifs.

Once a new motif is formed by adding a new fifth layer, the first layer and thus the first motif are dropped. The meaning of the symbols is described in the original paper (Michels-Clark et al., 2013).

\begin{tabular}{|c|c|c|c|c|c|c|c|c|c|}
\hline \multirow[b]{2}{*}{ From } & \multicolumn{9}{|l|}{ To } \\
\hline & $\ldots e b_{\mathrm{L}}$ & $\ldots e b_{\mathrm{R}}$ & $\ldots e e$ & $\ldots b_{\mathrm{L}} b_{\mathrm{R}}$ & $\ldots b_{\mathrm{L}} b_{\mathrm{L}}$ & $\ldots b_{\mathrm{L}} e$ & $\ldots b_{\mathrm{R}} b_{\mathrm{L}}$ & $\ldots b_{\mathrm{R}} b_{\mathrm{R}}$ & $\ldots b_{\mathrm{R}} e$ \\
\hline$e b_{\mathrm{L}}$ & 0 & 0 & 0 & $t-\Delta / 2$ & $c-\Delta / 2$ & $e_{1}+\Delta$ & 0 & 0 & 0 \\
\hline$e e^{\kappa}$ & $\left(1-e_{2}\right) / 2$ & $\left(1-e_{2}\right) / 2$ & $e_{2}$ & 0 & 0 & 0 & 0 & 0 & 0 \\
\hline$b_{\mathrm{L}} b_{\mathrm{R}}$ & 0 & 0 & 0 & 0 & 0 & 0 & $t$ & $c$ & $e_{1}$ \\
\hline$b_{\mathrm{L}} b_{\mathrm{L}}$ & 0 & 0 & 0 & $t$ & $c$ & $e_{1}$ & 0 & 0 & 0 \\
\hline$b_{\mathrm{L}} e^{\mathrm{L}}$ & $\left(1-e_{2}\right) / 2-\Delta$ & $\left(1-e_{2}\right) / 2+\Delta$ & $e_{2}$ & 0 & 0 & 0 & 0 & 0 & 0 \\
\hline$b_{\mathrm{R}}^{\mathrm{n}} e^{\mathrm{n}}$ & $\left(1-e_{2}\right) / 2+\Delta$ & $\left(1-e_{2}\right) / 2-\Delta$ & $e_{2}$ & 0 & 0 & 0 & 0 & 0 & 0 \\
\hline
\end{tabular}

affected and remain unchanged. The transition probabilities in Table 1 (rows 4, 5, 7, 8) and Fig. 2 (rows 1 and 2) of the original paper were different from those used in the numerical calculations. Corrected transition probabilities as used in the computations are given in Table 1 and Fig. 1 of this article. The $\Delta$ parameter in the stacking model expresses the preference for the fifth layer in a five-layer stack to be eclipsed with respect to the first layer. This statement corrects the original text on p. 1622, lines 4-7. In the original Fig. 2 the helicity of the layer stacks $b_{\mathrm{L}}$ and $b_{\mathrm{R}}$ in rows 3 and 4 had been given as opposite to those in rows 1, 2 and 5. Fig. 1 of this article shows rows 3 and 4 corrected to correspond to rows 1,2 and 5 .

\section{References}

Michels-Clark, T. M., Lynch, V. E., Hoffmann, C. M., Hauser, J., Weber, T., Harrison, R. \& Bürgi, H. B. (2013). J. Appl. Cryst. 46, $1616-1625$. 